\title{
A Report on Some Boundary Element Adventures
}

\author{
J.C.F. Telles \\ COPPE / Universidade Federal do Rio de Janeiro \\ Programa de Engenharia Civil, Caixa Postal 68506, \\ CEP 21945-970, Rio de Janeiro, Brazil
}

\begin{abstract}
The present paper discusses the author's experience and contributions to the implementation and development of the boundary element method (BEM). The main motivation for this descriptive text is to pay homage to Professor Frank Rizzo's wellknown career, dedicated to boundary integral techniques, whose recent retirement certainly deserves recognition of the BEM community. The present author feels very honoured for the opportunity to celebrate Prof. Rizzo's achievements and shares the feeling that his pioneer work undoubtedly has much to be thanked for.
\end{abstract}

\section{Introduction}

It is perhaps appropriate to introduce this celebration by presenting and commenting upon my own experiences in the formulation and development of this fascinating technique. To this end, it only seems natural that we start with the explanation of how and when my own BEM interest initiated and to follow from there into what, in my opinion, are my main contributions to BEM research.

A brief comparison between BEM in context to other methods is then followed by a presentation of the industrial applications I have been involved with in the course of my BEM investigations.

Rather than discussing my own views about the future of boundary elements the present author finds it more interesting to expand the text presenting some current subjects to which most of my recent research involvement has been concentrated on. A few figures with illustrative results are then presented to finalize this worthy deference to Professor Rizzo. The first application deals with Biot's consolidation theory for poro-elastic saturated media assuming incompressible grains and fluid, and adopts time-independent fundamental solutions [1]. The second [2] summarizes the applications of a numerical Green's function boundary element procedure which has been applied to solve a wide variety of linear elastic fracture mechanics problems. The formulation is based on the ability of the hypersingular boundary integral equation to represent potential/displacement discontinuities and is used to generate a numerical Green's function for the problem. Therefore, the existing cracks and obstructions of the potential continuity are embedded in the fundamental solution avoiding element discretization over such particular surfaces. 


\section{Historical Account of My BEM Interest}

Back in 1977, Professor Carlos Brebbia came to COPPE and presented a talk entitled "Is the Finite Element Method the Best Numerical Technique?" At that moment I was a recently contracted Lecturer who was doing research leading towards a doctoral degree. In those days we were all working with finite elements and, to the best of our knowledge, this also included him. We therefore expected the lecture to be a sound "yes". It was a great surprise to hear him say "no, it is the boundary integral equation or as we now call it, the boundary element method". I had heard of the technique before when in 1976 J.C. Lachat came to our institution to participate in a French-Brazilian Colloquium on Numerical Techniques, but until Carlos' presentation I was not really aware of the BEM potentiality. On that day, also based on the page-proofs of a Brebbia and Dominguez paper [3], I decided to study this "new" solution method and to give it a try. Needless to say this "try" was enough to convince me to take it further and soon enough I was reading the seminal works produced by Rizzo [4] and Cruse [5]. The final product of this first experience was the development of a 2-D linear element FORTRAN computer program for potential problems governed by the Laplace Equation and its elastostatics counterpart. In 1978, with that background, I went to the University of Southampton where I obtained a $\mathrm{PhD}$ degree in 1981. The subject of my doctoral research was the application of boundary elements to nonlinear material problems, including elastoplastic, visco-plastic and creep for 2-D applications. Much of this work can be found in the first book I wrote [6].

I believe the work on inelastic material problems was my first BEM contribution I can consider important, but there were other topics, such as the use of alternative fundamental solutions [7] (e.g. half-plane), singular and quasi-singular numerical integration schemes $[8,9]$, consistent boundary element formulations with equilibrium satisfaction [10], nonlinear elasto-dynamics [11], boundary and finite element combination [10,12], application to cathodic protection problems [13], hyper-singular formulations [14] and fracture mechanics [2] which also contributed to the development of the boundary element technique.

Generally speaking, the BEM is without doubt a powerful numerical technique which has already established itself within the scientific community, especially the direct formulation which proves to be the most reliable of the boundary integral approaches. As to the finite element and finite difference methods, they are both well-known procedures which together with the BEM can be used by engineers and scientists to solve practical or more scientific oriented problems. It is this author's opinion that there are many problems which are better solved by BEM, but by all means not all of them and there is plenty of room not only for these three methods but also to include more recently developed techniques such as the so-called meshless alternatives.

As for industrial applications, there are many practical areas which apply (or can apply) the results of the BEM research work. I can underline my two major applied areas of interaction with industry. I believe the subject of cathodic protection has been the most known: together with colleagues of mine, we have developed a BEM computer system called PROCAT for the Brazilian State Owned Oil Company PETROBRAS [13]. It started in the early eighties and the project also involved researchers of the Material and 
Metallurgical Department of COPPE. This long lasting project greatly contributed to our understanding of the electrochemical phenomenon of corrosion. Another important interaction with industry has been with Furnas, a Brazilian state company responsible for the production and transmission of electric energy in the south-eastern part of Brazil. This interaction started in the nineties and resulted in a BEM computer system, CAV3D, specially developed [15] to analyze the rock excavations of the hydraulic circuit of the Serra da Mesa Hydroelectric Power Plant in the north of Brazil.

\section{Topics of Current Involvement}

Among my recent research work activities, I would like to point out the application of BEM to solve problems governed by Biot's consolidation theory. In this application the poro-elastic saturated media is dealt with using a time marching scheme that applies finite differences to integrate the diffusion equation and the total volumetric stresses. Each phase of the consolidation phenomenon is solved independently within a time step and the exchange of common data provides means of coupling the two analyses [1]. It is worth mentioning that domain integrals in the elastostatic integral equation are retained to provide simple means of simulating media heterogeneity and nonlinear material behaviour, such as plasticity. A removal of this domain cell discretization can be later effected using existing alternative techniques.

A typical result obtained by the adopted Biot's solution strategy can be seen in Fig. 1.

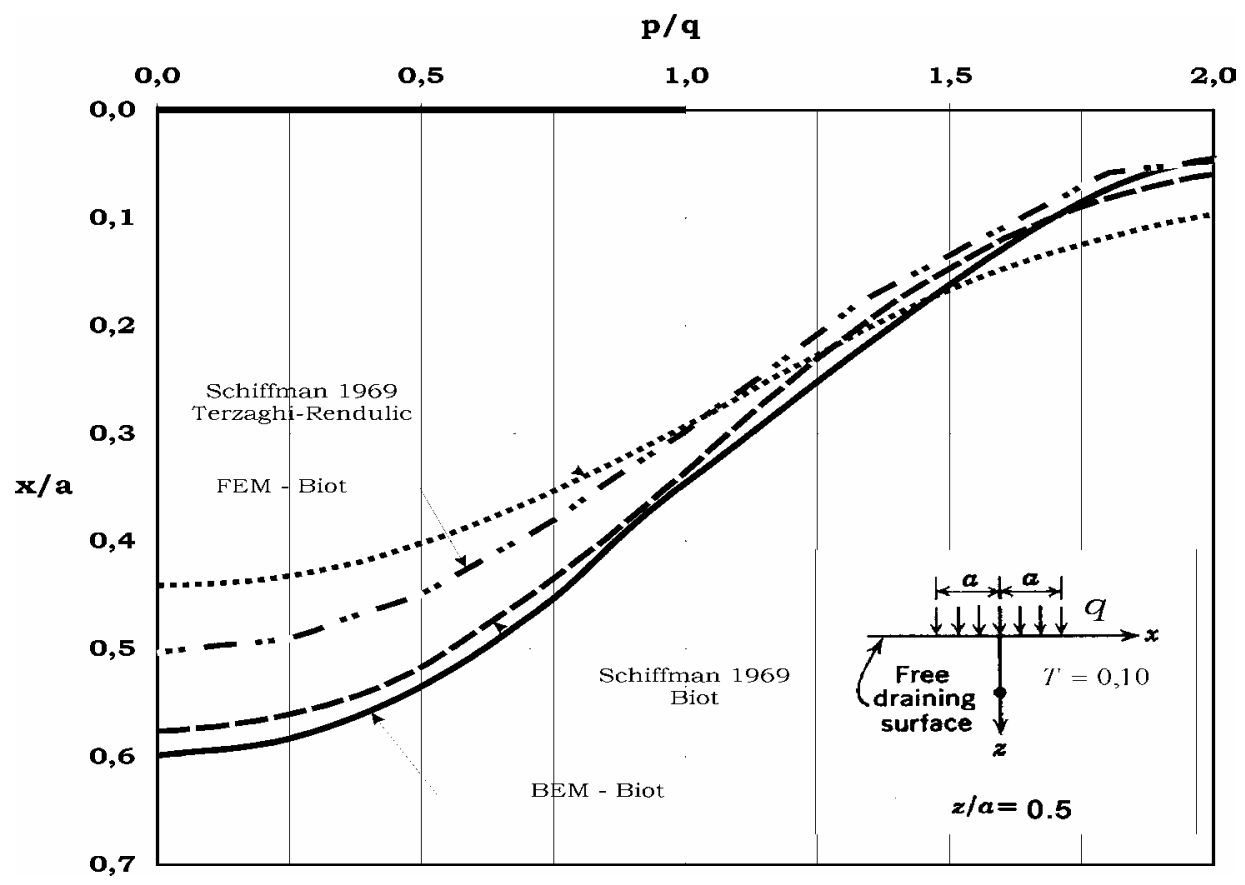

Figure 1. Normalized excess pore-pressures - horizontal distribution for an adjusted time factor of 0.10 for a depth of half the load semi-width 
Another subject that has been developed is the implementation, to various fracture mechanics or potential discontinuity problems, of a numerical Green's function boundary element approach. In the Numerical Green's Function (NGF) formulation, the hyper-singular boundary integral equation is used to generate a Green's function solution to the particular potential discontinuity surface or fracture geometry involved. Such a Green's function is then implemented, as the fundamental solution, in a standard BEM computer package in which element discretization of actual crack surfaces is no longer required [16].

An illustrative result obtained with the NGF formulation is depicted in Fig. 2. The example consists of a plane pressure wave acting in the normal direction upon a flat crack embedded in an infinite medium. The problem has been solved for a wide variety of frequencies and the computed stress intensity factor is normalized with respect to the corresponding static solution [17].

$\mathrm{KI} / \mathrm{K} 0$

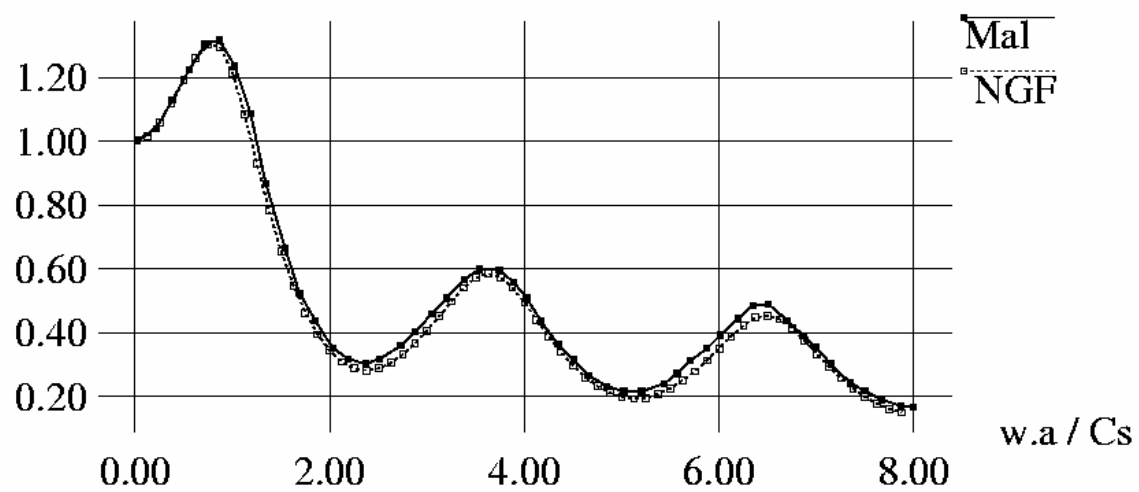

Figure 2. Normalized stress intensity factor for alternative frequencies of incoming wave.

\section{Conclusions}

The author would like to close this short presentation thanking, once again, Professor Rizzo whose academic career has been an inspiration to us all.

\section{References}

1. Cavalcanti, M.C., Telles, J.C.F, Biot's consolidation theory - application of BEM with time independent fundamental solutions for poro-elastic saturated media, Engineering Analysis with Boundary Elements, in press, 2002.

2. Telles, J.C.F., Castor, G.S., Guimarães, S., “A Numerical Green's Function Approach for Boundary Elements Applied to Fracture Mechanics. Int. J. Num. Meth. Engg., 38, p. 3259-3274, 1995. 
3. Brebbia, C.A., Dominguez, J. The Boundary Element Method for Potential Problems, Appl. Math. Modelling, 1, p. 372-378, 1977.

4. Rizzo, F.J., An Integral Equation Approach to Boundary Value Problems of Classical Elastostatics, Q. Appl. Math., 25, p. 83-95, 1967.

5. Cruse, T.A., Numerical Solutions in Three Dimensional Elastostatics, Int. J. Solids Structures, 5, p. 1259-1274, 1969.

6. Telles, J.C.F., The Boundary Element Method Applied to Inelastic Problems, Lecture Notes in Engineering 1, Springer-Verlag, Berlin, 1983.

7. Telles, J.C.F., Brebbia, C.A., Boundary Element Solution for Half-Plane Problems, Int. J. Solids Structures, 17, p. 1149-1158, 1981.

8. Telles,J.C.F., A Self-Adaptive Co-ordinate Transformation for Efficient Numerical Evaluation of General Boundary Element Integrals, Int. J. Num. Meth. Engineering, 24, p. 959-973, 1987.

9. Telles, J.C.F., Oliveira, R.F., Third Degree Polynomial Transformation for Boundary Element Integrals: Further Improvements, Engineering Analysis with Boundary Elements, 13, p. 135-141, 1994.

10. Telles, J.C.F., De Paula, F.A., Boundary Elements with Equilibrium Satisfaction -A Consistent Formulation for Potential and Elastostatic Problems, Int. J. Num. Meth. Engineering, 32,p. 609-621, 1991.

11. Telles, J.C.F., Carrer, J.A.M., A Boundary Element Formulation to Solve Transient Dynamic Elastoplastic Problems, Computer and Structures, 45, p. 707-713, 1992.

12. De Paula, F.A., Telles, J.C.F., A Comparison Between Point Collocation and Galerkin for Stiffness Matrices Obtained by Boundary Elements, Engineering Analysis with Boundary Elements, 6, p. 123 - 128, 1989.

13. Telles, J.C.F., Mansur, W.J., Wrobel, L.C., Marinho, M.G., Numerical Simulation of a Cathodically Protected Semi-Submersible Platform Using the PROCAT System, Corrosion Journal, 46, p. 513-518, 1990.

14. Guimarães, S., Telles, J.C.F., On the Hyper-Singular Boundary Element Formulation for Fracture Mechanics Applications, Engineering Analysis with Boundary Elements, 13, p. 353-363, 1994.

15. Franco, J.A.M, Assis, A.P., Mansur, W.J., Telles, J.C.F., Santiago, J.A.F. Design Aspects of the Underground Structures of the Serra da Mesa Hydroelectric Power Plant, Int. J. Rock Mech. and Min. Sci, 34, p. 763-772, 1997.

16. Castor, G.S., Telles, J.C.F., The 3-D BEM Implementation of a Numerical Green's Function for Fracture Mechanics Applications, Int. J. Num. Meth. Engineering, 48, p. 1199-1214, 2000.

17. Barra, L.P.S. and Telles, J.C.F. A Hypersingular Numerical Green's Function Generation for BEM Applied to Dynamic SIF Problems, Egineering Analysis with Boundary Elements, 23, p. 77-87, 1999. 